\title{
Caracterização climatológica da severidade de secas do Estado do Ceará - Brasil ${ }^{1}$
}

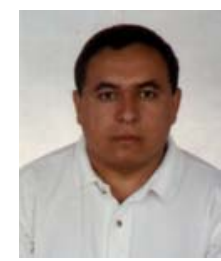

Tarcisio da S. Barra ${ }^{2}$, José M. N. da Costa ${ }^{3}$, Tantravahi V. R. Rao ${ }^{4}$, Gilberto C. Sediyama ${ }^{5}$, Williams P. M. Ferreira ${ }^{6} \&$ Francisco S. Dantas Neto ${ }^{7}$

\footnotetext{
1 Parte da Dissertação de Mestrado do primeiro autor, apresentada ao curso de Pós-Graduação em Meteorologia Agrícola da Universidade Federal de Viçosa

2 DEA/UFV. Av. P.H. Rolfs s/n., Viçosa, MG. Fone: (31) 3899-2729, Fax: (31) 3891-2735 (Foto)

${ }^{3}$ DEA/UFV. Fone: (31) 3899-1898, Fax: (31) 3899-2735. E-mail: jmncosta@ufv.br

4 DCA/UFCG. Campina Grande, PB. E-mail: ramana@dca.ufpb.br

5 DEA/UFV. Fone: (31) 3899-1905, Fax: (31) 3899-2735. E-mail: sediyama@ufv.br

${ }^{6}$ DEA/UFV. Fone: (31) 3899-2729, Fax: (31) 3899-2735. E-mail:cfsouza@ufv.br

7 DEA/UFV. Fone: (31) 3899-2729, Fax: (31) 3899-2735. E-mail:fsolon@alunos.ufv.br
}

Protocolo 095 - 27/6/2001

Resumo: O objetivo deste trabalho foi caracterizar as secas do Estado do Ceará, com base no índice de severidade de seca de Palmer. Foram utilizadas séries históricas de dados pluviométricos e de temperatura do ar de 21 localidades desse Estado, fornecidas, respectivamente, pela Superintendência de Desenvolvimento do Nordeste - SUDENE, e pela Fundação Cearense de Meteorologia e Recursos Hídricos - FUNCEME. Foi constatado a ocorrência de secas com diferentes graus de severidade, no Estado do Ceará, sendo as secas moderadas e severas as mais freqüentes.

Palavras-chave: índice de severidade de seca de Palmer, climatologia

\section{Climatological characterization of the drought severity in the State of Ceará - Brazil}

\begin{abstract}
The objective of this work was to characterize the droughts in the State of Ceará based on the Palmer drought severity index. Historical data series of rainfall and air temperature of 21 localities of this State, were provided by the "Superintendência de Desenvolvimento do Nordeste - SUDENE", and by the "Fundação Cearense de Meteorologia e Recursos Hídricos FUNCEME", respectively. The occurrences of droughts of different degrees of severity were verified in the State of Ceará, with frequent moderate and severe droughts.
\end{abstract}

Key words: Palmer drought severity index, climatology

\section{INTRODUÇÃO}

O Estado do Ceará que cobre uma área de $146.348 \mathrm{~km}^{2}$ está situado na região Nordeste do Brasil. A grande variabilidade anual da precipitação causa problemas de seca de diferentes intensidades no Estado do Ceará.

A seca é um fenômeno climático que ocorre em uma região quando a precipitação, para determinado período de tempo, apresenta valores muito abaixo do normal climatológico. Embora não exista uma definição universal para a seca, esse fenômeno está sempre associado à deficiência hídrica, podendo ser avaliada em termos de sua duração, intensidade e variação espacial. Segundo McNab \& Karl (1991) a dificuldade de se apresentar uma definição geral para seca deve-se à necessidade de especificar os componentes do ciclo hidrológico afetados pela deficiência hídrica e o período de tempo a ela associado. Várias definições têm sido propostas para caracterizar a severidade, a duração e a variação espacial desse fenômeno, justificando os diferentes enfoques utilizados na análise: meteorológico, hidrológico, agrícola e socioeconômico.

Uma técnica amplamente utilizada na caracterização das secas é baseada nos índices de seca, que consistem em equações com variáveis climáticas e procedimentos estatísticos para determinar a intensidade, a duração e a freqüência com que essa anomalia ocorre. Tal procedimento foi utilizado, por vários pesquisadores, para caracterizar as secas na Região Nordeste do Brasil (Xavier \& Xavier, 1984; Kumar et al., 1989; Lima et al., 1989; Repelli \& Nobre, 1991; Azevedo \& Silva, 1994; Freitas, 1996). Todavia, em nenhum desses trabalhos foi utilizado o índice de severidade de seca de Palmer na avaliação da severidade das secas do Estado do Ceará. Esse índice de seca tem sido utilizado com sucesso na caracterização da severidade das secas nos Estados Unidos(Palmer, 1965), na Austrália (Kothavala, 1999), no Brasil (Assis et al., 1997), no 
Canadá (Akinremi et al., 1996) e na Europa (Briffa et al., 1994). Nesse contexto, o presente trabalho teve como objetivo analisar a variação espacial e temporal do índice de severidade de seca de Palmer, no Estado do Ceará.

\section{MATERIAL E MÉTODOS}

Foram utilizadas séries históricas de dados mensais de temperatura e precipitação pluvial de 21 localidades. A altitude dos postos pluviométricos considerados neste trabalho variou entre $15 \mathrm{~m}$ (Jaguaruna) e $885 \mathrm{~m}$ (Ibiapina). A temperatura média diária varia entre 22 a $33{ }^{\circ} \mathrm{C}$. A precipitação média anual está em torno de $775 \mathrm{~mm}$. Esses dados foram fornecidos pela Superintendência de Desenvolvimento do Nordeste - SUDENE, e pela Fundação Cearense de Meteorologia e Recursos Hídricos FUNCEME. A Tabela 1 apresenta as coordenadas geográficas, a duração e o número de anos da série de dados pluviométricos das 21 localidades incluídas neste trabalho.

Utilizaram-se dados mensais de temperatura do ar e de precipitação pluvial para se calcular o balanço hídrico mensal, e o modelo de solo de duas camadas, proposto por Palmer (1965) assumindo-se uma capacidade de armazenamento de água da camada superficial de $25 \mathrm{~mm}$, enquanto a capacidade de água disponível da camada inferior dependerá das características do solo de cada localidade.

$\mathrm{Na}$ determinação da evapotranspiração potencial, Palmer (1965) utilizou o método de Thornthwaite, na versão proposta em 1948. Segundo Alley (1984) a estimativa da evapotranspiração potencial pela equação de Thornthwaite representa uma limitação do método proposto por Palmer (1965).

equação de Thornthwaite não apresentou resultados satisfatórios para o Estado do Ceará, conforme foi constatado por Barra \& Dantas Neto (1998), em uma comparação de métodos de estimativa de evapotranspiração potencial para o Estado do Ceará. Assim, a estimativa da evapotranspiração potencial pelo método de Thornthwaite foi substituída pelo método de Hargreaves \& Samani (1985) de acordo com a seguinte equação:

$$
\mathrm{ETo}=0,0023 \mathrm{Q}_{\mathrm{o}}\left(\mathrm{T}_{\max }-\mathrm{T}_{\min }\right)^{0,5}(\mathrm{~T}+17,8)
$$

em que:

$$
\begin{array}{ll}
\mathrm{ET}_{0} & \text { - evapotranspiração potencial, } \mathrm{mm} \mathrm{d}^{-1} \\
\mathrm{Q}_{\mathrm{o}} & \text { - radiação extraterrestre, } \mathrm{mm} \mathrm{d} \mathrm{d}^{-1} \\
\mathrm{~T}_{\max } & \text { - temperatura máxima diária do } \mathrm{ar},{ }^{\circ} \mathrm{C} \\
\mathrm{T}_{\min } & \text { - temperatura mínima diária do } \mathrm{ar},{ }^{\circ} \mathrm{C} \\
\mathrm{T}^{\mathrm{O}} & \text { - temperatura média diária do ar, }{ }^{\circ} \mathrm{C}
\end{array}
$$

A perda de água da camada superficial $\left(\mathrm{L}_{\mathrm{s}}\right)$ e da camada inferior $\left(\mathrm{L}_{\mathrm{u}}\right)$ foram calculadas pelas seguintes equações:

$$
\begin{gathered}
\left.\mathrm{L}_{\mathrm{s}}=\mathrm{S}_{\mathrm{s}} \text { ou PE - P (o que for menor }\right) \\
\mathrm{L}_{\mathrm{u}}=\left(\mathrm{PE}-\mathrm{P}-\mathrm{L}_{\mathrm{s}}\right) \frac{\mathrm{S}_{\mathrm{u}}^{\prime}}{\mathrm{AWC}} \operatorname{para~}_{\mathrm{u}} \leq \mathrm{S}_{\mathrm{u}}{ }_{\mathrm{u}}
\end{gathered}
$$

em que:

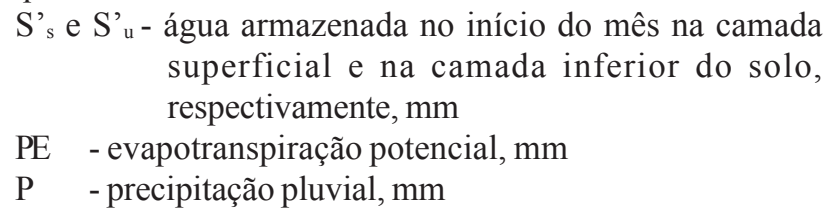

AWC - capacidade de água disponível nas duas camadas de solo, $\mathrm{mm}$

Os termos recarga potencial (PR), perda potencial de água no solo (PL) e escoamento superficial potencial (PRO) são derivados do balanço hídrico mensal e utilizados na determinação do índice de seca. A PR que representa a quantidade de umidade necessária para o solo atingir a capacidade de campo, foi expressa pela seguinte equação:

$$
\mathrm{PR}=\mathrm{AWC}-\mathrm{S}^{\prime}
$$

Tabela 1. Coordenadas geográficas das estações pluviométricas no Estado do Ceará

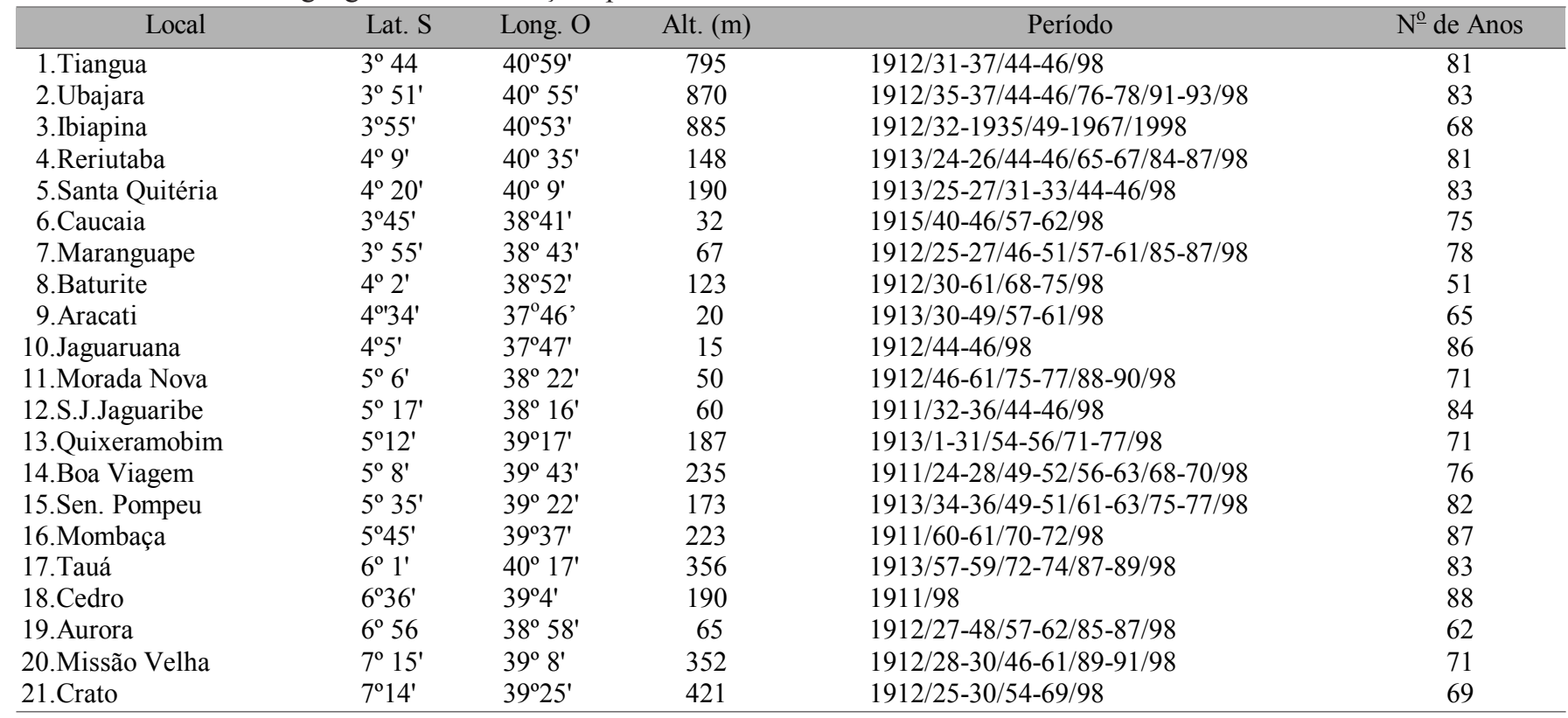


em que:

S' - quantidade de água disponível em ambas as camadas do solo, no início do mês, assumindo-se que a camada superficial tem $25 \mathrm{~mm}$ de capacidade de água disponível enquanto a capacidade de água disponível da camada inferior será determinada com base nas características de solo de cada localidade.

A perda potencial de água no solo (PL) que representa a quantidade de água no solo e que poderá ser perdida por evapotranspiração, assumindo-se que não ocorreu precipitação no referido mês, foi expressa pela seguinte equação:

$$
\mathrm{PL}=\mathrm{PL}_{\mathrm{s}}+\mathrm{PL}_{\mathrm{u}}
$$

em que:

$\mathrm{PL}_{\mathrm{s}}$ - perda potencial de água na camada superficial do solo, $\mathrm{mm}$

$\mathrm{PL}_{u}$ - perda potencial de água na camada inferior do solo, mm

O cálculo de $\mathrm{PL}_{\mathrm{s}} \mathrm{e} \mathrm{PL}_{\mathrm{u}}$ foi feito com base nas seguintes equações:

$$
\mathrm{PL}_{\mathrm{s}}=\mathrm{PE} \mathrm{ou} \mathrm{S}_{\mathrm{s}}{ }_{\mathrm{s}} \text { (o que for menor) }
$$

$$
\mathrm{PL}_{\mathrm{u}}=\left(\mathrm{PE}-\mathrm{PL}_{\mathrm{s}}\right) \frac{\mathrm{S}_{\mathrm{u}}^{\prime \prime}}{\mathrm{AWC}}
$$

O escoamento superficial potencial (PRO) que representa o total de água armazenada disponível no solo, foi calculado pela seguinte equação:

$$
\mathrm{PRO}=\mathrm{AWC}-\mathrm{PR}=\mathrm{S}^{\prime}
$$

Os termos definidos anteriormente pelas Eqs. 4 a 8, foram calculados para cada mês, em cada localidade, com base no balanço hídrico mensal. Com base nos valores médios mensais desses termos, determinaram-se quatro coeficientes adimensionais $\left(\alpha_{\mathrm{i}}, \beta_{\mathrm{i}}, \gamma_{\mathrm{i}}\right.$ e $\left.\delta_{\mathrm{i}}\right)$ para cada mês, em cada localidade, conforme as seguintes equações:

$$
\begin{gathered}
\alpha_{i}=\frac{\overline{\mathrm{ET}}}{\overline{\overline{\mathrm{PE}}}} \\
\beta_{\mathrm{i}}=\frac{\overline{\mathrm{R}}}{\overline{\overline{\mathrm{PR}}}} \\
\gamma_{\mathrm{i}}=\frac{\overline{\mathrm{RO}}}{\overline{\overline{\mathrm{PRO}}}}=\frac{\overline{\mathrm{RO}}}{\mathrm{S}^{\prime}} \\
\delta_{\mathrm{i}}=\frac{\overline{\mathrm{L}}}{\overline{\mathrm{PL}}}
\end{gathered}
$$

em que:

$$
\alpha_{i} \quad \text { - coeficiente de evapotranspiração para o mês "i" }
$$

$\beta_{\mathrm{i}} \quad$ - coeficiente de recarga para o mês " $i$ "

$\gamma_{\mathrm{i}} \quad$ - coeficiente de escoamento superficial para o mês "i"

$\delta_{\mathrm{i}} \quad$ - coeficiente de perda de água do solo para o mês "i"

ET - evapotranspiração atual calculada, $\mathrm{mm}$

$\mathrm{R}$ - recarga (ganho líquido de umidade do solo durante determinado período), $\mathrm{mm}$

RO - escoamento superficial estimado, $\mathrm{mm}$

i - meses do ano: $i=1,2, \ldots, 12$

(一) - valor médio mensal dos componentes com base na série histórica disponível para cada localidade

Os coeficientes $\alpha_{i}, \beta_{i}, \gamma_{i}$ e $\delta_{\mathrm{I}}$, foram utilizados no cálculo dos valores "CAFEC", que representam o valor particular de um parâmetro que seria climaticamente apropriado para as condições do tempo e do lugar que está sendo analisado. As condições climaticamente apropriadas, serão calculadas para os seguintes componentes: precipitação, evapotranspiração atual, recarga, escoamento superficial e perdas de água no solo usando-se as seguintes equações:

$$
\begin{gathered}
\hat{\mathrm{ET}}=\alpha \mathrm{PE} \\
\hat{\mathrm{R}}=\beta \mathrm{PR} \\
\hat{\mathrm{RO}}=\gamma \mathrm{PRO} \\
\hat{\mathrm{L}}=\delta \mathrm{PL}
\end{gathered}
$$

As Eqs. 13 a 16 foram combinadas para estimar a quantidade de precipitação necessária em um mês para atender as demandas médias de evapotranspiração, escoamento superficial e água armazenada no solo, com base em condições de umidade antecedente expressa pela seguinte equação:

$$
\hat{P}=\hat{E T}+\hat{R}+\hat{R O}-\hat{L}
$$

A deficiência ou excesso hídrico, foi calculada pela seguinte equação:

$$
\mathrm{d}=\mathrm{P}-\hat{\mathrm{P}}
$$

em que:

d - deficiência hídrica (-) ou excesso (+), mm

P - precipitação do mês considerado, $\mathrm{mm}$

$\mathrm{P}$ - precipitação calculada para o referido mês, $\mathrm{mm}$

O índice de anomalia de umidade (Z), proposto por Palmer (1968), foi expresso como:

$$
\mathrm{Z}=\mathrm{d} \mathrm{k}
$$

em que:

k - fator de ponderação proposto por Palmer (1965), calculado a partir da série histórica de dados de cada localidade. 

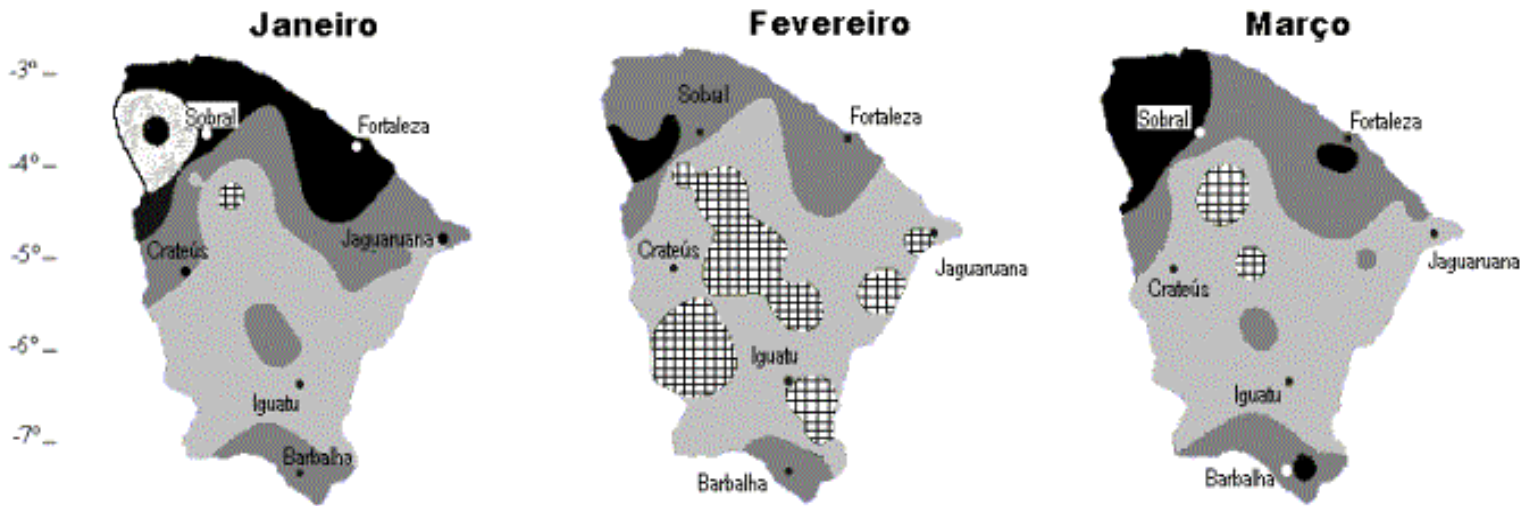

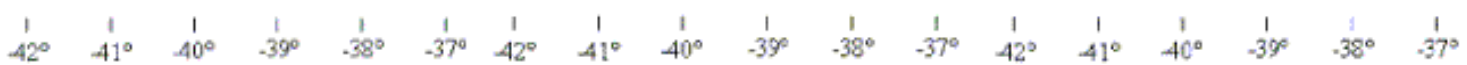
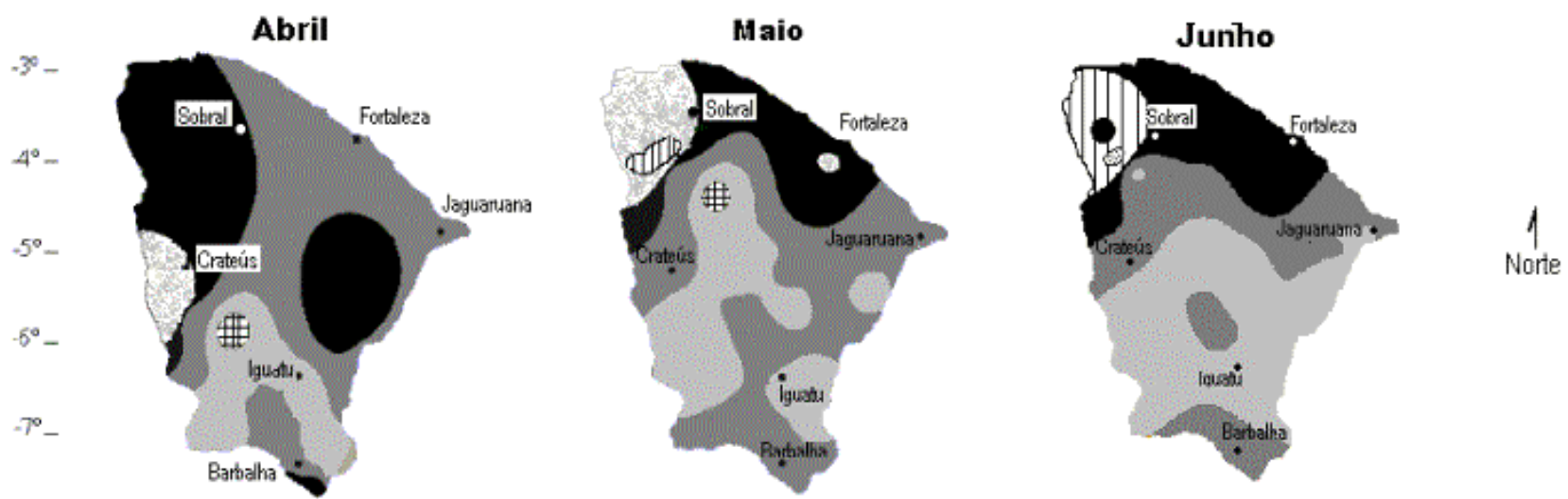

$\begin{array}{cccccccccccccccccc}1 & 1 & 1 & 1 & 1 & 1 & 1 & 1 & 1 & 1 & 1 & 1 & 1 & 1 & 1 & 1 & 1 & 1 \\ 42^{\circ} & -41^{\circ} & -40^{\circ} & -39^{\circ} & -38^{\circ} & -37^{\circ} & 42^{\circ} & -41^{\circ} & -40^{\circ} & -39^{\circ} & -38^{\circ} & -37^{\circ} & 42^{\circ} & -41^{\circ} & -40^{\circ} & -39^{\circ} & -38^{\circ} & 37^{\circ}\end{array}$
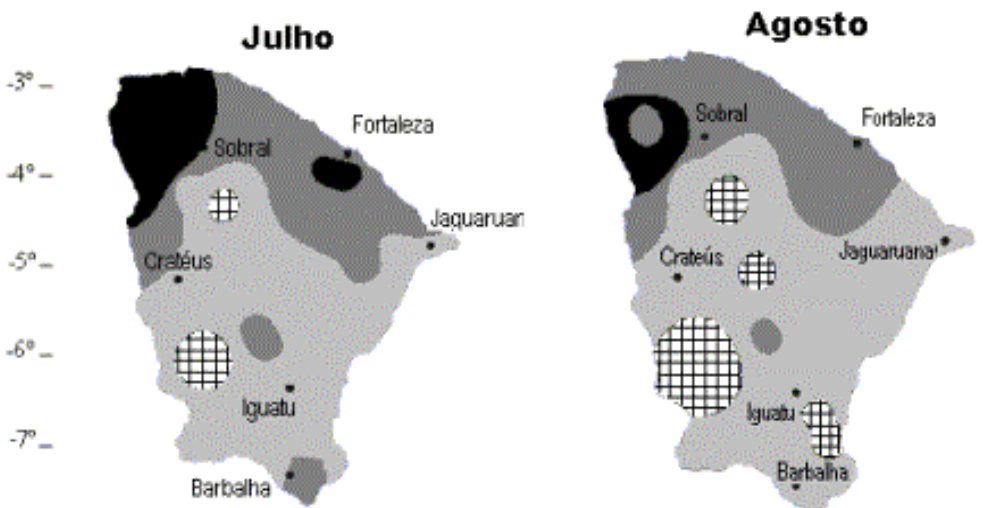

Setembro

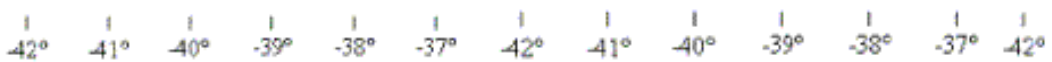
Outubro

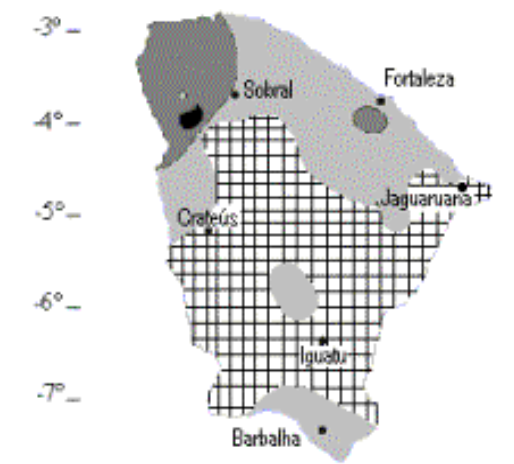

Novembro

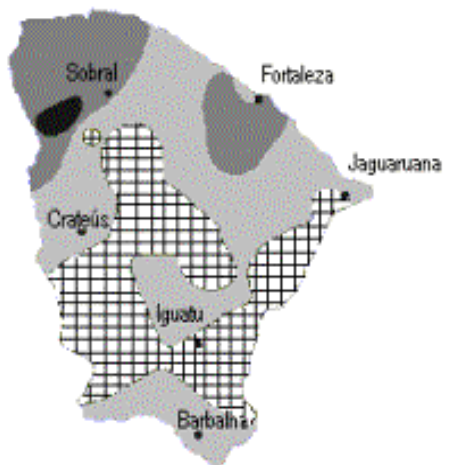

\section{$\begin{array}{ccccc}1 & 1 & 1 & 1 & 1 \\ 47^{\circ} & -40^{\circ} & -39^{\circ} & -38^{\circ} & -37^{\circ}\end{array}$ \\ Dezembro}
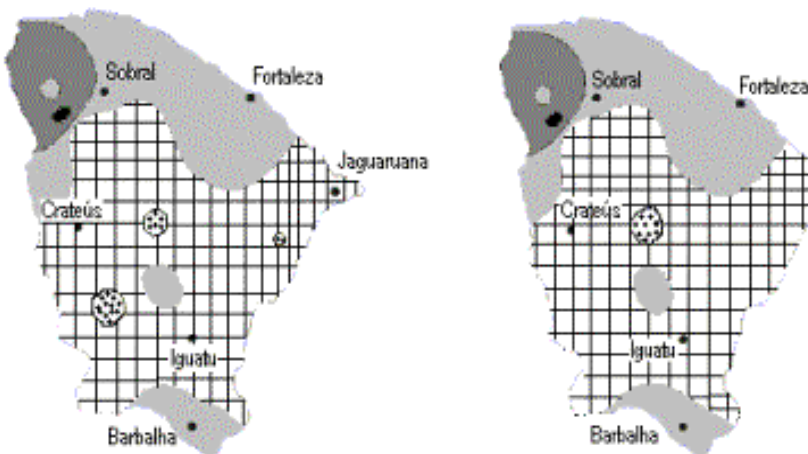

$\begin{array}{cccccccccccccccccc}1 & 1 & 1 & 1 & 1 & 1 & 1 & 1 & 1 & 1 & 1 & 1 & 1 & 1 & 1 & 1 & 1 & 1 \\ 42^{\circ} & 41^{\circ} & 40^{\circ} & -39^{\circ} & -38^{\circ} & -37^{\circ} & -42^{\circ} & 41^{\circ} & -40^{\circ} & -39^{\circ} & -38^{\circ} & -37^{\circ} & -42^{\circ} & 41^{\circ} & 40^{\circ} & -39^{\circ} & -38^{\circ} & -37^{\circ}\end{array}$

Figura 1. Variação espacial dos valores médios mensais do índice de severidade de seca de Palmer (ISSP) no Estado do Ceará 
Após calcular o índice "Z" para determinado mês ( i ), o índice de severidade de seca de Palmer (ISSP) foi calculado pela seguinte equação:

$$
\operatorname{ISSP}_{i}=\operatorname{ISSP}_{i-1}+Z_{i} / 3-0,103 \operatorname{ISSP}_{i-1}
$$

Os valores do ISSP variam de extremamente úmido $(>4)$ a seca extrema $(<-4)$. Os valores de ISSP entre 0,99 e $-0,99$ representam condições próximas ao normal, conforme mostra a Tabela 2. A variação espacial do ISSP foi feita com o uso do "software" Idrisi, versão 2.0 para Windows, utilizando-se os dados de 21 localidades do Estado do Ceará.

Tabela 2. Classificação das condições secas e úmidas com base nos valores do ISSP

\begin{tabular}{cl}
\hline Índice (ISSP) & \multicolumn{1}{c}{ Classificação } \\
\hline$\geq 4,00$ & Extremamente úmido \\
3,00 a 3,99 & Muito úmido \\
2,00 a 2,99 & Moderadamente úmido \\
1,00 a 1,99 & Ligeiramente úmido \\
0,99 a $-0,99$ & Aproximadamente normal \\
$-1,00$ a $-1,99$ & Ligeiramente seco \\
$-2,00$ a $-2,99$ & Seca moderada \\
$-3,00$ a $-3,99$ & Seca severa \\
$\leq-4,00$ & Seca Extrema \\
\hline
\end{tabular}

\section{RESULTADOS E DISCUSSÃO}

A variação espacial dos valores médios mensais do ISSP no Estado do Ceará está ilustrada na Figura 1. No mês de janeiro, predomina a seca moderada, atingindo cerca de $45 \%$ da área do Estado, compreendendo as microrregiões do sertão, sudeste e sudoeste. Nesse mês a ocorrência de valores de ISSP correspondentes à condição ligeiramente seca, predomina em cerca de 30\% da área do Estado, localizada principalmente na parte sul e numa faixa que se prolonga desde o litoral da zona Jaguaribana até a parte oeste. A ocorrência de seca severa nesse mês foi insignificante, abrangendo uma área inferior a $1 \%$.

No mês de fevereiro, embora represente o início da estação chuvosa, caracteriza-se pela incidência de seca moderada, em praticamente metade da área do Ceará, abrangendo uma parte do litoral e quase todas as suas microrregiões. Nesse mês, a incidência do ISSP referente à condição ligeiramente seca atinge $25 \%$ da área do Estado, abrangendo a microrregião do litoral, oeste e sul do Estado. A incidência de seca severa nesse mês é expressiva, atingindo, em média, $22 \%$ da área do Estado, distribuídas em áreas localizadas a leste, centro e oeste.

A incidência de seca moderada na estação chuvosa do Ceará foi maior em março, abrangendo $53 \%$ da área do Estado e atingindo principalmente as partes sudeste, sudoeste e sertão central. A condição ligeiramente seca também atingiu, nesse mês, uma área significativa de aproximadamente 30\% do Estado. Todavia, constatou-se grande redução na incidência de seca severa, em relação ao mês anterior, atingindo uma área de aproximadamente $3 \%$ do Estado.

No mês de abril há predominância da condição ligeiramente seca, atingindo cerca de $41 \%$ da área do Ceará, distribuída no litoral, leste, centro e sul do Estado. A ocorrência da condição
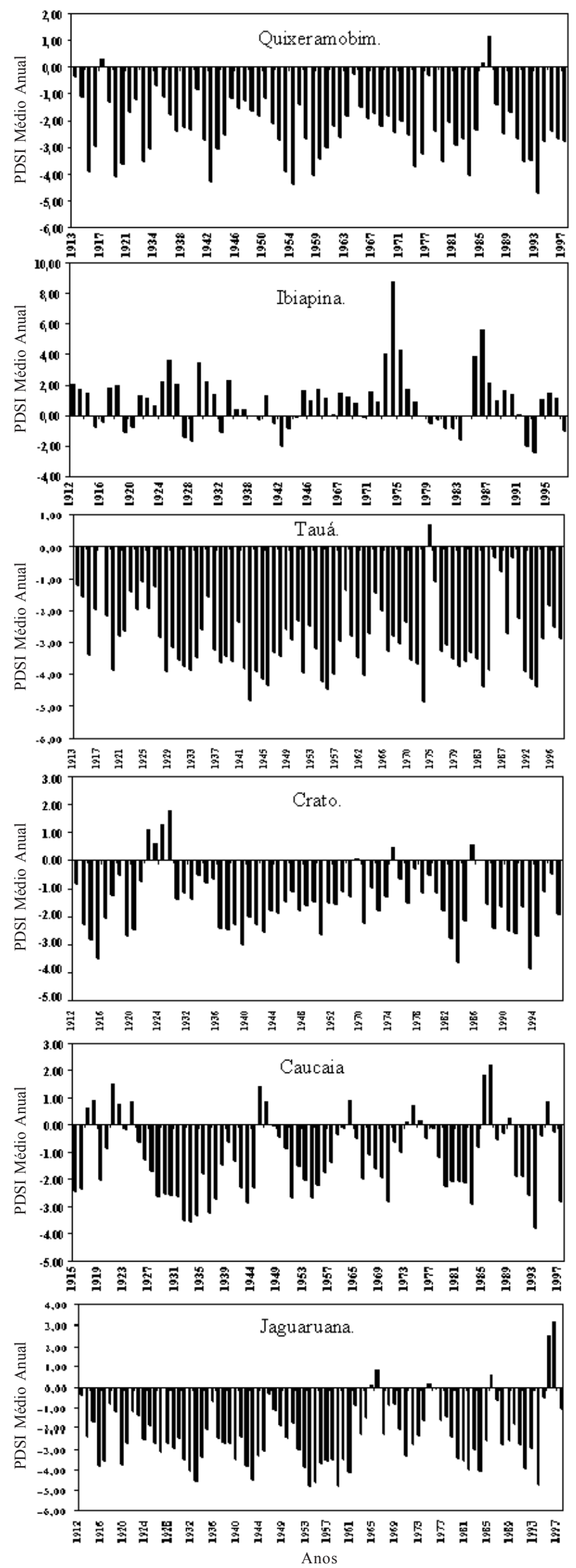

Figura 2. Valores médios anuais do índice de severidade da seca de Palmer (ISSP) para seis localidades do Estado do Ceará 
Tabela 3. Classificação dos anos muito secos no Estado do Ceará associados aos valores de ISSP para seis localidades

\begin{tabular}{|c|c|c|c|c|c|c|c|c|c|c|c|c|}
\hline \multirow{3}{*}{ Ano } & \multicolumn{12}{|c|}{ Localidade } \\
\hline & \multicolumn{2}{|c|}{ Jaguaruana } & \multicolumn{2}{|c|}{ Quixeramobim } & \multicolumn{2}{|c|}{ Tauá } & \multicolumn{2}{|c|}{ Crato } & \multicolumn{2}{|c|}{ Caucaia } & \multicolumn{2}{|c|}{ Ibiapina } \\
\hline & Chuva & ISSP & Chuva & ISSP & Chuva & ISSP & Chuva & ISSP & Chuva & ISSP & Chuva & ISSP \\
\hline 1915 & 161 & $-3,83$ & 209 & $-3,92$ & 478 & $-3,41$ & 684 & $-3,5$ & 411 & $-2,41$ & 585 & $-0,79$ \\
\hline 1919 & 165 & $-3,80$ & 16 & $-4,12$ & 227 & $-3,89$ & 441 & $-2,71$ & 592 & $-2,05$ & 494 & $-1,10$ \\
\hline 1932 & 175 & $-4,57$ & 294 & $-3,52$ & 290 & $-3,87$ & & & 646 & $-3,53$ & 609 & $-1,15$ \\
\hline 1942 & 269 & $-4,47$ & 356 & $-4,28$ & 293 & $-4,83$ & 636 & $-2,56$ & 633 & $-2,29$ & 701 & $-2,03$ \\
\hline 1953 & 195 & $-4,78$ & 329 & $-3,90$ & 542 & $-3,18$ & 728 & $-1,13$ & 961 & $-2,05$ & & \\
\hline 1993 & 118 & $-4,74$ & 240 & $-4,71$ & 372 & $-4,38$ & 568 & $-3,85$ & & $-3,82$ & 950 & $-2,48$ \\
\hline Média $^{*}$ & 727 & $-2,35$ & 756 & $-2,29$ & 583 & $-2,88$ & 1059 & $-1,47$ & 1215 & $-1,21$ & 1599 & 0,89 \\
\hline
\end{tabular}

* Média anual da série histórica de dados

próxima ao normal também foi expressiva em cerca de $38 \%$ da área do Estado. A incidência de seca severa nesse mês também foi insignificante, em cerca de 1\% da área do Estado. Em maio, embora a seca moderada seja predominante, corresponde aproximadamente $42 \%$ da área do Estado, a incidência da seca moderada também é expressiva, atingindo uma área de cerca de $28 \%$ do Estado. A incidência de seca severa nesse mês é praticamente inexpressiva. $\mathrm{O}$ mês de junho marca o início do predomínio da seca moderada, atingindo cerca de 39\% da área do Estado. Nesse mês, embora a incidência de seca severa não seja expressiva, a ocorrência das condições ligeiramente seca e próxima do normal corresponde, respectivamente, a uma área de 30 e $23 \%$ do Estado.

A partir de julho evidencia-se o aumento na incidência de seca moderada e de seca severa no Ceará. Agosto é o mês que apresenta maior incidência da seca moderada, atingindo 58\% do Estado, seguido pelo mês de setembro, também com grande porcentagem de seca moderada e severa.

Quanto à variação espacial dos valores médios do ISSP no último trimestre do ano se constata, seguindo a tendência dos meses anteriores, máxima ocorrência de seca severa no mês de novembro, com cerca de $60 \%$. A partir do mês de dezembro, que caracteriza a pré-estação chuvosa, verifica-se grande redução na ocorrência de secas severas.

A variação intra-anual do índice de severidade de seca de Palmer, para seis localidades representativas das microrregiões do Ceará, está apresentada na Figura 2. Com exceção de Ibiapina, todas as localidades se caracterizam pela ocorrência de secas, com diferentes graus de severidade.

A Tabela 3 destaca os anos classificados como muito secos em mais de 75\% do Estado do Ceará, de 1900 até 1979, conforme estudo realizado por Xavier e Xavier (1984) para as referidas seis localidades, além do ano de 1993, que foi bastante seco. Em todas as localidades houve grande redução no total de precipitação anual, em relação à média dos dados anuais de toda a série, porém se observa, com base no ISSP, grande variação na severidade das secas entre essas localidades. Nas localidades de Jaguaruana (Vale do Jaguaribe), Quixeramobim (Sertão Central) e Tauá (Sertão Sudoeste) prevaleceram as condições de seca severa e extrema; enquanto na localidade de Crato, ao sul do Estado, e Caucaia, no litoral, ocorreu predominância de seca moderada, e em Ibiapina foi observada apenas a condição ligeiramente seca. Esses resultados revelam a potencialidade de uso de índices de seca, como o ISSP, na caracterização da severidade e dos impactos das secas, de forma muito mais eficiente que a simples utilização de dados de chuva.

\section{CONCLUSÕES}

Os resultados encontrados permitiram as seguintes conclusões:

1. A ocorrência de secas nas localidades estudadas do Estado do Ceará variou com o grau de severidade, sendo secas moderadas e severas as mais freqüentes, excetuando-se as localidades das microrregiões serranas.

2. A variação espacial da severidade das secas do Estado do Ceará pode ser caracterizada pelo índice de severidade de seca de Palmer, mostrando a sua utilidade na avaliação dos impactos das secas no Estado, o que não seria possível apenas com a análise dos dados de precipitação.

\section{LITERATURA CITADA}

Akinremi, O.O.; McGinn, S.M.;Barr, A.G. Evaluation of the Palmer Drought Index on the Canadian Praires. Journal of Climate, Boston, v.9, n.5, p. 897-905, 1996.

Alley, W.M. The Palmer drought severity index: Limitations and assumptions. Journal of Climate and Applied Meteorology, Boston, v.23, n.7, p.1100-1109, 1984.

Assis, F.N.; Martins, S.R.; Mendez, M.E.G. Índice de severidade de seca de Palmer no Rio Grande do Sul. In: Congresso Brasileiro de Agrometeorologia, 10, 1997, Piracicaba. Anais... Piracicaba: SBAGRO, 1997. p.383-385.

Azevedo, P.V.; Silva, V.P.R. Índice de seca de Bhalme e Mooley: uma adaptação regional. In: Congresso Brasileiro de Meteorologia, 8, 1995, Belo Horizonte. Anais... Belo Horizonte: SBMET, 1994. p.696-699.

Barra, T.S.; Dantas Neto, F.S. Avaliação de métodos para a estimativa da evapotranspiração de referência no Estado do Ceará. In: Congresso Brasileiro de Meteorologia, 10, 1998, Brasília. Anais... Brasília: SBMET, 1998. p.57-69.

Briffa, K.; Jones, P.; Hulme, M. Summer moisture availability across Europe, 1892-1991: An analysis based on the Palmer drought severity index. International Journal of Climatology, Sheffield, n.14, p.457-506, 1994.

Freitas, M.A.S. Previsão de secas por meio de métodos estatísticos e redes neurais e análise de suas características através de diversos índices (Ceará - Nordeste do Brasil). In: Congresso Brasileiro de Meteorologia, 9, 1996, Campos do Jordão. Anais...Campos do Jordão: SBMET, 1996. p.163-165.

Hargreaves, G.H.; Samani, Z.A.; Reference crop evapotranspiration from temperature. Applied Engineering in Agriculture, St. Joseph, v.1, n.2, p.96-99, 1985. 
Kothavala, Z. The duration and severity of drought over eastern Australia simulated by a coupled ocean-atmosphere GCM with a transient increase in $\mathrm{CO}_{2}$. Environmental Modelling and Software, New York, n.14, p.243-252, 1999.

Kumar, K.K.; Silva, J.A.T.; Silva, B.B. Estudo de seca e aridez em Fortaleza, CE. In: Congresso Brasileiro de Agrometeorologia, 6, 1989, Maceió. Anais... Maceió: SBAGRO, 1989. p.175-184.

Lima, M.C.; Santos, R.C.; Moraes, J.C. Estudo de secas no Estado de Alagoas. In: Congresso Brasileiro de Agrometeorologia, 6, 1989, Maceió. Anais... Maceió: SBAGRO, 1989. p.160-169.

McNab, A.L.; Karl, T.R. Climate and droughts. In: Paulson, R. W.; Chase, E.B.; Roberts, R.S.; Moody, D.W. (eds.) Compilers, national water summary 1988-89 Hydrologic events and floods and droughts. Reston, Virginia: U.S. Geological Survey. 1991.p.89-98.
Palmer, W.C. Meteorological drought. Washington: U.S. Weather Bureau, 1965. 65p.

Palmer, W.C. Keeping track of crop moisture conditions, nationwide: The new crop moisture index. Weatherwise, Washington, v.21, n.4, p.156-161, 1968.

Repelli, C.A.; Nobre, C.A. Um novo índice de anomalia de precipitação para o Nordeste do Brasil. In: Congresso Brasileiro de Agrometeorologia, 7, 1991, Viçosa. Anais... Viçosa: SBAGRO, 1991.p.21-25.

Xavier, T.M.B.S.; Xavier, A.F.S. Classificação de anos secos e chuvosos na Região Nordeste do Brasil e sua distribuição espacial. In: Congresso Brasileiro de Meteorologia, 3, Belo Horizonte. Anais... Belo Horizonte: SBMET, 1984. p.267-275. 